\title{
Self Field Measurements by Hall Sensors on the SeCRETS Long Sample CICCs in SULTAN
}

\author{
Yu. A. Ilyin, A. Nijhuis, H. H. J. ten Kate, and P. Bruzzone
}

\begin{abstract}
The aim of this work is to determine the existence and degree of the current unbalance of two types of Cable-In-Conduit Conductors (CICC) of the SeCRETS long sample experiment, and its influence on the conductors' performance. The self-field measurements are performed by using six sets of annular Hall sensors, each containing six sensors, and two linear arrays with ten sensors. The change of the self-field is associated with the redistribution of the transport current between the strands inside the conductor during and after a ramp of current, due to changes of the applied magnetic field or temperature of the conductor. During the DC, AC losses and stability tests, the signals from the Hall sensors were recorded. In DC tests, a clear change of the self-field pattern is observed in the high field region when either current or temperature approached their critical $\left(I_{c s}\right.$ and $\left.T_{c s}\right)$ values. No change in the self-field pattern is observed in the experiments with pulsed fields. The method requires improvements for a reasonable quantitative assessment of the current unbalance in the conductor.
\end{abstract}

Index Terms-Cable-In-Conduit superconductors, current distribution, self-field measurements.

\section{INTRODUCTION}

$\mathbf{T}$ HE MAIN reason for studying nonuniformity in the current distribution is that it may affect the performance of a cabled conductor at transient [1] or even quasi steady state conditions. In order to study the occurrence and possible impact of current unbalance, a self-field measurement method with Hall sensors (HS) is integrated in the SeCRETS experiment in the SULTAN facility at CRPP.

The main objective of the SeCRETS experiment [2] is to assess the impact of segregated copper wires on the transient field stability of $\mathrm{Nb}_{3}$ Sn CICCs. Two CICCs, identical in the overall cross sections except for the distribution of the copper stabilizer, either fully included in the superconducting strands or partly segregated as copper wires, are tested. The sample layout is performed as a bifilar, one layer solenoid with a diameter of $350 \mathrm{~mm}$ and 22 turns, consisting of two conductor sections A (without segregated copper) and B (with segregated copper) connected in series at one end into a hairpin joint [2].

Self-field measurements are performed at eight locations during DC and transient operation runs. The selected HSs

Manuscript received September 24, 2001. This work was supported in part by the EU under Contract EFDA-00/552.

Yu. A. Ilyin, A. Nijhuis, and H. H. J. ten Kate are with the University of Twente, Faculty of Applied Physics, 500AE Enschede, the Netherlands (e-mail y.ilyin@tn.utwente.nl).

P. Bruzzone is with CRPP-Technologie de la Fusion, CH-5232 Villigen-PSI, Switzerland (e-mail: pierluigi.bruzzone@psi.ch).

Publisher Item Identifier S 1051-8223(02)04261-6.

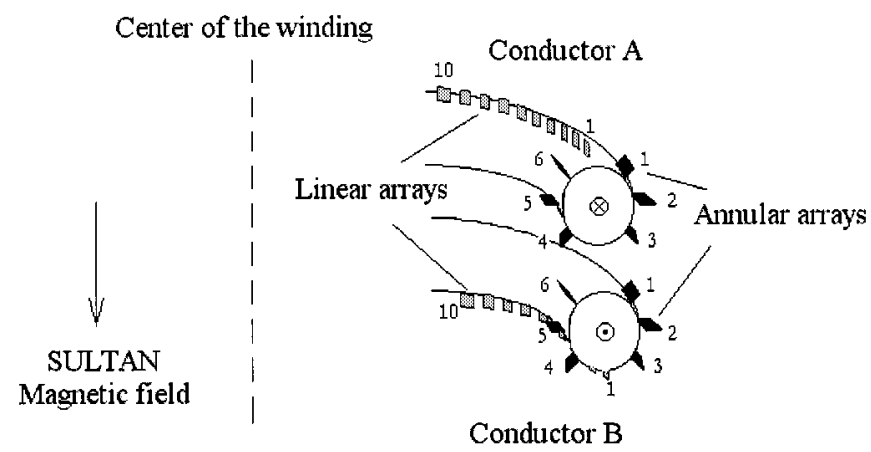

Fig. 1. Arrangement of the HSs in relation to the conductors and the direction of the magnetic field from SULTAN.

combine a good geometrical resolution with the ability to operate in DC as well as AC mode. The current distribution is studied under the following conditions:

- steady state current distribution at different DC settings,

- "slow" current redistribution during the DC tests (approaching the cable $T_{c s}$ ) at high background field,

- presence and propagation of boundary induced coupling currents (BICCs) beyond the conductor section subjected to the pulsed field,

- fast evolution of the current distribution, close to the pulsed field zone and far away from it, after a pulsed field applied in the range close to the quench/no-quench threshold.

The results of the measurements and their analysis are presented and discussed.

\section{INSTRUMENTATION}

In total 58 miniature HSs were attached to the SeCRETS conductors. Six sensors were lost during the installation and after cool-down. The sensors size is either $1.5 \times 1.5 \mathrm{~mm}$ or $2 \times 2 \mathrm{~mm}$, the active area is $0.2 \mathrm{~mm}^{2}$. The sensors have been delivered with their calibration sheets.

Six "annular arrays" of HSs ( 3 for each conductor) are placed respectively just after the hairpin joint, at the peak field location and at the pulse coils location. Each array contains 6 HSs at $60^{\circ}$ spacing, close to the outer surface of the conductor jacket (Fig. 1). Two "linear arrays," each containing 10 sensors, are placed along the conductor in the high field, evenly distributed over a full cable pitch of $160 \mathrm{~mm}$ (Fig. 1). All sensors are oriented in such a way that they measure the tangential component of the self-field. More details can be found in [2]. 


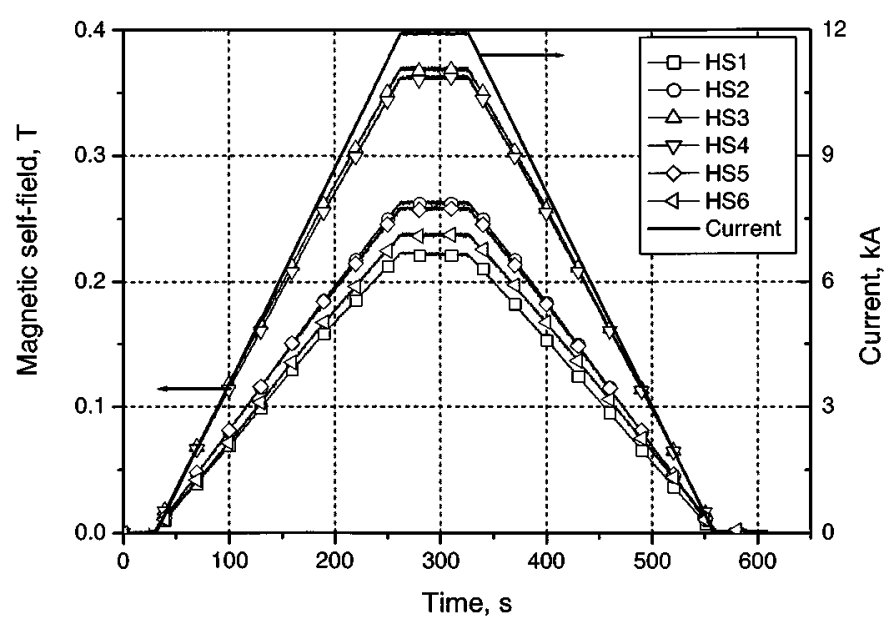

Fig. 2. Typical time dependence of the magnetic field with current as measured by HSs in the annular array of conductor B near the joint.

\section{RESULTS}

\section{A. Analysis of the HS Signals During the AC Runs}

In the $\mathrm{AC}$ loss runs, the aim of the self-field measurements is to detect BICCs. When only a section of a superconductor is exposed to an AC field, the BICCs may propagate in the adjacent sections of the conductor [3]. In the SeCRETS conductors, applying an AC field with the largest operating current of $12 \mathrm{kA}$, no change of the self-field pattern could be observed in the HSs placed next to pulsed coils. Only a very week $(25 \mathrm{mT})$ signal is observed, which is identified as being directly induced by the magnetic field from pulsed coil. Hence, no BICCs could be observed. The lack of detectable BICCs is in agreement with the relatively low level of interstrand coupling loss [2].

The self-field pattern from the HSs next to pulsed coils has been monitored during transient field shots (full wave) followed by either a recovery or quench. The pulsed sinusoidal field (one period) of $1 \mathrm{~T}$ maximum amplitude is applied to the conductors being in a $10 \mathrm{~T}$ background field with a transport current of $10 \mathrm{kA}$. No detectable change of the current distribution is observed between the pulse field and the subsequent current decrease during $\sim 300 \mathrm{~ms}$. Assuming that the local field at the pulsed coils actually exceeds $B_{c}$ during a full wave shot, the "disturbance" is perfectly homogeneous and no detectable current redistribution occurs.

\section{B. Analysis of the HS Signals During the DC Runs}

As an example, a typical DC run for conductor B is shown in Fig. 2. Traces from the annular HS array near the joint are plotted for the case of $8 \mathrm{~T}$ background field and $12 \mathrm{kA}$. The field values can be represented as a polar plot in which the angle corresponds to the annular position of a HS. The magnitude of the radius is the magnetic field value monitored by a sensor at a chosen time, mostly at maximum transport current (Fig. 3).

In any array the HSs are sensitive not only to the local selffield of the conductors, but also to the magnetic field from the neighboring conductors, as well as to background magnetic field from SULTAN. At zero current in the SeCRETS winding, the nonzero offset of a HS signal due to SULTAN field and other effects (e.g., thermal voltages) was subtracted.

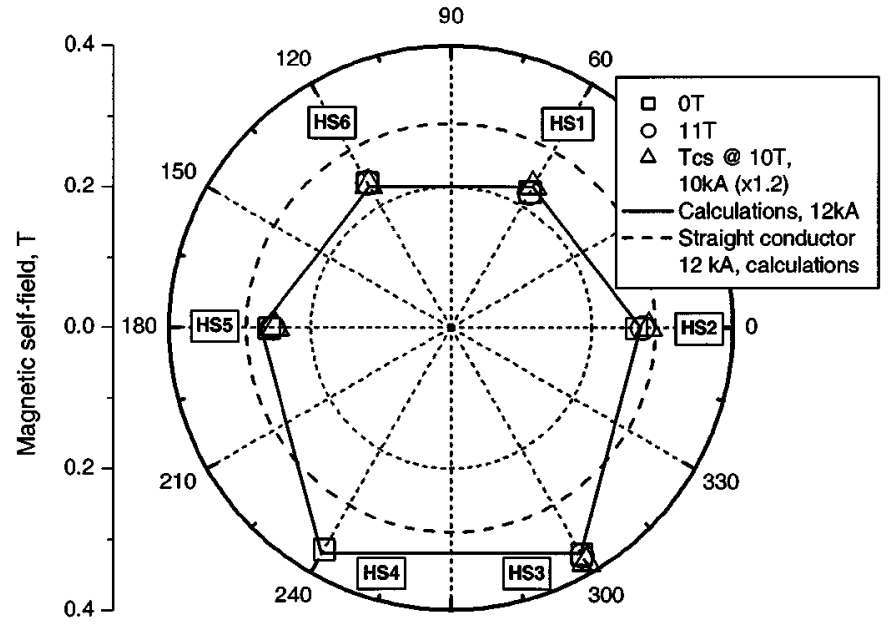

Fig. 3. Measured and calculated (for a homogeneous current distribution) magnetic field at the annular HS arrays near the joint for conductor B. The experimental points are evaluated at the maximum current. Solid lines connect the calculated (supposing uniform current distribution) values of the self-field at the location of HSs. The bold dashed circle represents the magnetic field pattern around an infinitively long conductor with the same current and without neighboring conductors.

The field contribution from the neighboring conductors, proportional to the operating current, is calculated at each HS of the annular and linear arrays with the Biot-Savar expression. The winding geometry is simplified to a set of circular turns and the current in the neighboring turns is assumed to be uniform over the conductors' cross-section. The apparent difference between the signals of the sensors in the same array [up to $40 \%$ in the annular arrays (Fig. 2)] is principally due to the nonhomogeneous field contribution from the neighboring conductors and to the curvature of the turns.

The response of the annular array of conductor B close to joints is shown in Fig. 3. The experiments were done at different levels of magnetic field and at a maximum transport current of $12 \mathrm{kA}$ at $50 \mathrm{~A} / \mathrm{s}$. The HS signals at the temperature above the $T_{C S}$ of the superconductor are also shown in the same plot. Some of the runs were performed with a current of $10 \mathrm{kA}$. In order to compare them with other runs made at $12 \mathrm{kA}$, the measured values were multiplied by a factor of 1.2.

The average noise level increases from $\pm 0.6 \%$ at $I_{T}=12 \mathrm{kA}$ and $B_{D C}=0 \mathrm{~T}$ up to $\pm 1.5 \%$ at $11 \mathrm{~T}$. The maximum deviation of the calculated self-field magnitudes (for homogeneous current distribution) from the measured ones at $B_{D C}=0 \mathrm{~T}$ is $\sim 4 \%$. Besides the possible nonuniform current distribution, this difference may be partly attributed to the accuracy of the HS positioning (at least $\pm 0.2 \mathrm{~mm}$ ), deviation of the HS calibration coefficient due to thermal cycling, parasitic thermal voltages from the soldered connections and, in lesser extent, to simplifications in the calculations.

A change of the measured self-field magnitude after compensation is observed when varying the background magnetic field (and temperature). The maximum deviation between the experimental data at $0 \mathrm{~T}$ and $11 \mathrm{~T}$ for conductor A amounts to $5 \%$ (average 3\%) and for conductor B 3\% (average 1.2\%). This difference can be due to: a change in the current distribution, the nonlinearity of the HS calibration coefficient (from $0 \mathrm{~T}$ to $2 \mathrm{~T}$ his can already amount to $2 \%$ ) and the increased noise level 


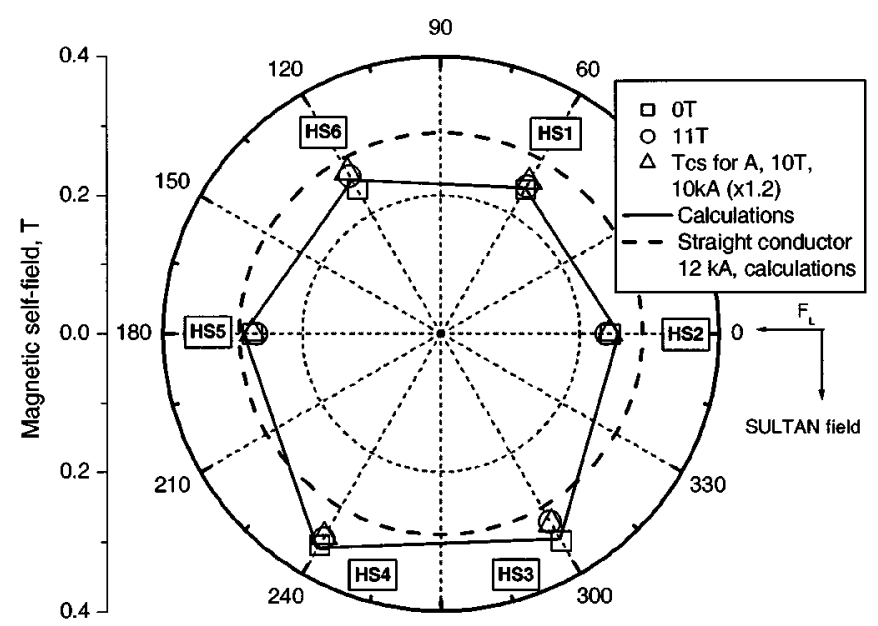

Fig. 4. Measured and calculated (for homogeneous current distribution) magnetic field at the annular HS array for conductor A near the pulsed coils at different background fields and close to $T_{C S}$. The direction of the SULTAN magnetic field and Lorentz forces are indicated.

at high magnetic field levels from SULTAN, which may affect the error bar.

In Fig. 4 the measured and calculated values (for homogeneous current distribution) of the self-field around conductor A are shown for the annular array near the pulsed coils. The data presented in Fig. 4 are obtained in the same experimental run as given above for the joint array.

Due to their orientation with respect to the direction of the SULTAN field, which is somewhat below the maximum here, these HSs capture a considerable fraction of the background field. Therefore the signal-to-noise ratio becomes worse and amounts to $\pm 5 \%$ at $B_{D C}=0$ to $11 \mathrm{~T}$. The maximum deviation between the calculated (for homogeneous distribution) and measured values of self-field magnitudes at $B_{D C}=0 \mathrm{~T}$ and $12 \mathrm{kA}$ is within 5\%. The difference in HS signals with increasing magnetic field (and temperature) is larger than what is found for the array near the joint as presented in Fig. 3: $\sim 9 \%$ for conductor A (average $4.3 \%$ ) and $\sim 7 \%$ for conductor B (average $3.2 \%$ ). This time the difference can be caused by: a change in the current distribution, deformation of the strands bundle inside the conduit due to Lorentz force, larger error bar due to high noise level at high magnetic fields and the nonlinearity of the HS coefficient with increasing field, parasitic planar effect. It is not clear to which extent each possible source contributes to the deviation with respect to the current nonuniformity.

The signals from the annular arrays in the "high field" region change the most with increasing magnetic field (see Fig. 5, conductor A only). The maximum deviation between the calculated values for a homogeneous distribution and the measured ones at $B_{D C}=0 \mathrm{~T}$ amounts to $10 \%$. The bigger deviation at this location compared to the others can partly be attributed to the error of the self-field calculation (regarding the choice of how many full turns to take into account for the calculation), though this error is still considered to be low enough.

With increasing magnetic field (or temperature), the maximum deviation between the experimental data at $B_{D C}=0 \mathrm{~T}$ and $11 \mathrm{~T}$ amounts to $25 \%$ (average 13\%) for conductor $\mathrm{A}$ and $\sim 9 \%$ (average 5\%) for conductor B.

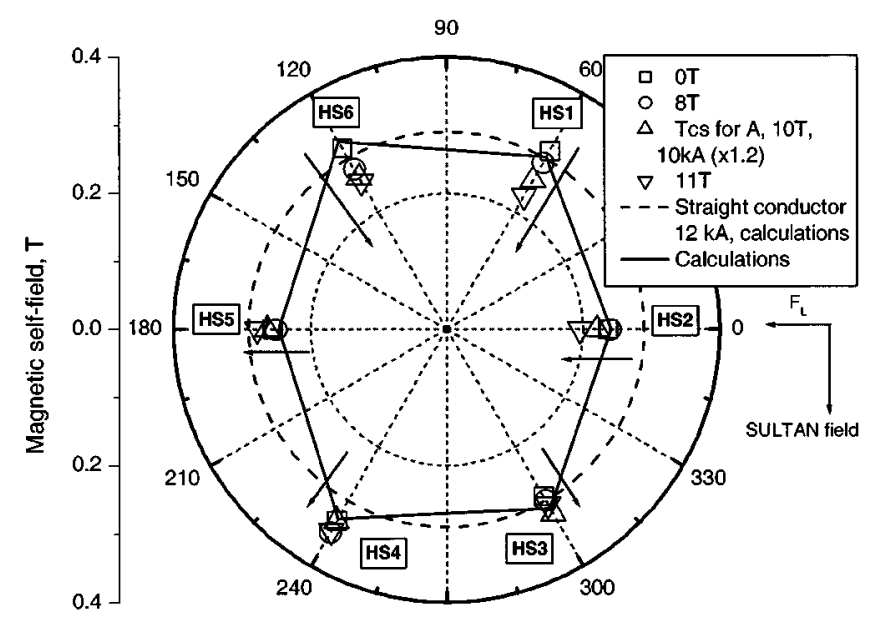

Fig. 5. Measured and calculated (for homogeneous current distribution) magnetic field at the annular HS arrays for conductor A in the high field region at different background fields and close to $T_{C S}$. The direction of the SULTAN magnetic field and Lorentz forces are indicated.

The average noise level at $B_{D C}=0-8 \mathrm{~T}$ is about $\pm 6 \%$ but at $11 \mathrm{~T}$ it increased significantly and a quantitative analysis of the results becomes somewhat speculative (see section C).

It was found that the deviations of the signals between $B_{D C}=0 \mathrm{~T}$ and $11 \mathrm{~T}$ are more severe in the case of conductor A. For conductor A the signals from HS1, 2, and 6 are decreasing and for the other sensors they are increasing with inclining magnetic field (see Fig. 5).

Besides the possible effects partly playing a role in causing such a pattern already mentioned, there is also another reason, which can lead to the change of the self-field pattern. If the conductor reaches the current sharing mode, the developed longitudinal voltage along the strands in the conductor will show a periodic pattern. The combination of the cable self-field and the applied SULTAN field causes a field transient across the cable cross-section. As one side of the cross section becomes saturated, this may eventually lead to a redistribution of the current to strands in the nonsaturated zone. The interstrand contact resistance determines the magnitude of the transverse currents inside the cable. Although it is conceivable that this self-field effect plays a role in the shift of the self-field pattern observed in Fig. 5, the interstrand contact resistance is probably too high for a large effect. The larger self-field change and shift of the current center, observed in conductor A, correlate well with the electric field observed at the high field section, ten times larger than in conductor B. The earlier voltage rise in conductor A for the same operating temperature is due to the higher compressive strain (inwards Lorentz forces) and therefore reduced critical current.

A similar correlation between high field voltage drop and change of the self field signal is observed in a $T_{C S}$ run, slowly increasing the temperature of the conductor up to a quench as shown in Fig. 6. During the same run small changes in the linear HS signals, up to $\sim 4 \%$ are observed during the temperature rise. The change of the HS signals in this case is less than observed in Fig. 5, most probably due to existence of a small-saturated zone already presented at the start of the temperature rise. 


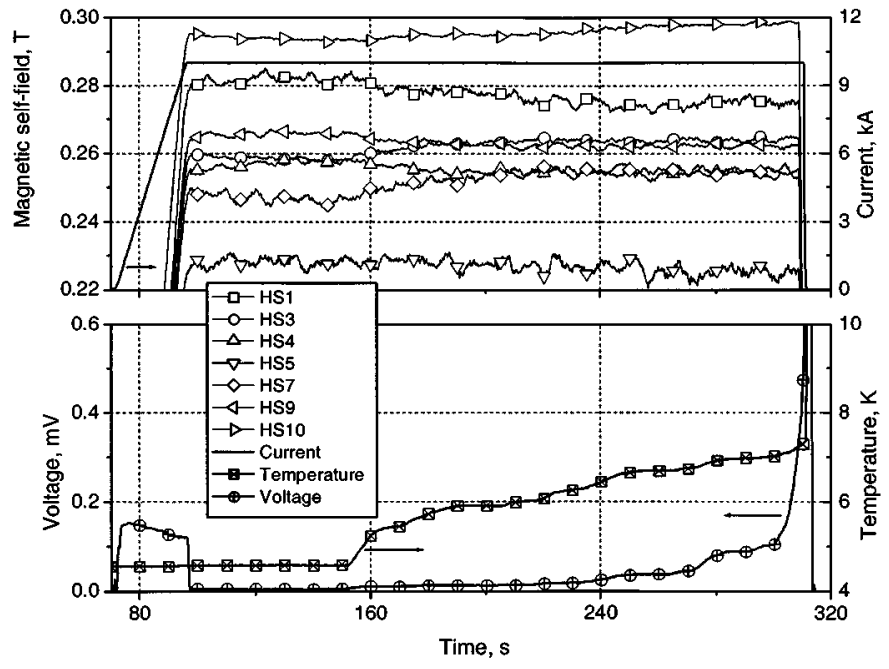

Fig. 6. Measured magnetic self-field from the linear HS (selected), voltage and temperature of conductor B in the high field region, while the temperature approaches $T_{c s}$.

Besides the effect of current redistribution approaching the current sharing mode, the transient phenomenon is also observed at zero magnetic field. During a ramp of transport current, the currents distribute between the strands mainly depending on their impedances. At constant transport current, the resistive part of the impedance becomes dominant and determines the current distribution.

In Fig. 7 the evolution in time of the signals from the linear Hall sensors near conductor $\mathrm{A}$ is shown during constant current after a ramp up to $12 \mathrm{kA}$ with $50 \mathrm{~A} / \mathrm{s}$. The change in amplitude of the signals is only $\sim 1-2 \%$. A similar effect is noticed in the experiments with a current ramp rate of $400 \mathrm{~A} / \mathrm{s}$. The characteristic time constants are in a range of 40-60 s for different Hall sensors. The change in the HS signals is associated with a slow current redistribution in the resistive mode or partly with the decay of induced loops. In the following section, the change of the HS signals is correlated to a redistribution of the current, modeled as sub-bundle currents.

\section{Analytical Model}

An inverse problem must be solved in order to calculate the unknown currents in the conductor cross section by using the known magnetic self-field distribution around the conductor. Because only six HS are available in each annular array, the model is developed for an array of six unknown currents distributed as identical angular segments in the conductor. The variables in the model are the six sub-bundle currents (and possibly the angle between the sensor array and current array). The known values are the magnetic fields at each sensor location and the total current in the conductor. The set of equations was solved with the help of Mathcad ${ }^{\mathrm{TM}}$ software. The model is explained more in detail in [4].

It is found that the error in the evaluation of the current distribution strongly depends on the error of the magnetic field measurements. The model shows that an error of about $3 \%$ in the self-field measurements already leads to $\sim 22 \%$ error in the segment current calculation. As explained above the experimental

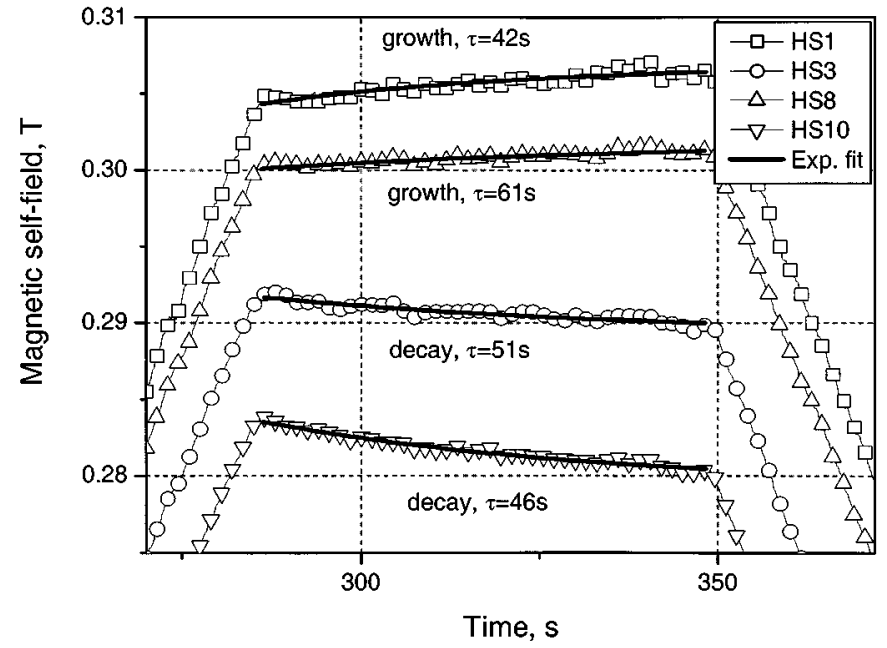

Fig. 7. Typical "flat" parts of the traces (selected) from the linear HS near conductor A, recorded at zero magnetic field and with current ramp rate of 50 $\mathrm{A} / \mathrm{s}$.

error in all cases is more than $3 \%$. Hence, it is impossible to determine the absolute degree of current unbalance in the conductor for stationary conditions. For this reason the accuracy of the method must be improved by roughly one order of magnitude. A significant improvement can already be achieved by a calibration of the field dependence of the HS coefficients.

Even better would be to calibrate the sensors after installation, avoiding the influence of geometrical errors.

However, it is possible to say something quantitatively about the dynamic behavior. For example, according to the model, a change in sensor signal of $\sim 4 \%$ in Fig. 6 corresponds to a change in the sub bundle current of $\sim 30 \%$. This means that the current in the sub bundle $(\sim 2 \mathrm{kA})$ changes roughly by $0.6 \mathrm{kA}$. For the case in Fig. 7 a change of the sensor signal of $\sim 2 \%$ corresponds to a change of the sub bundle current of roughly $0.3 \mathrm{kA}$.

\section{CONCLUSIONS}

From the analysis of HS's results, in AC mode, no change of the current distribution or induced currents is observed in the experiments with applied pulsed field.

In DC mode, no major unbalance has been observed in the current distribution over the cable cross section, either next to the joint or at high field. In current sharing mode (at high field region), a displacement of the current centerline is observed, possibly due to the self-field gradient over the conductor crosssection. The maximum change of the current in one of the six sub-bundles is estimated to be $0.6 \mathrm{kA}$.

The characteristic time constants of transient phenomenon are found in the range of 40-60 s for different HSs associated with the current changes in the sub bundles up to $0.3 \mathrm{kA}$.

A simple six-bundle (segment) model is proposed which shows that an error of about $3 \%$ in the self-field measurements leads to $22 \%$ error in the current calculation. For a quantitative assessment of current unbalance, the accuracy must be improved by up to one order of magnitude. 


\section{REFERENCES}

[1] V. S. Vysotsky, K. Funaki, and M. Takeo, "Current nonuniformity in multistrand superconducting cables-Experimental studies and its influence on stability of superconducting magnets," IEEE Trans. Appl. Superconduct., vol. 10 , no. 1 , pp. 351-357.

[2] P. Bruzzone, A. M. Fuchs, B. Stepanov, G. Vecsey, and E. Zapretilina, "Test results of SeCRETS, a stability experiment about segregated copper," IEEE Trans. Appl. Superconduct., vol. 11, no. 1, pp. 2018-2021.
[3] A. P. Verweij, "Electrodynamics of superconducting cables in accelerator magnets," Thesis, University of Twente, Enschede, 1994.

[4] A. Nijhuis, Y. Ilyin, and H. H. J. ten Kate, "Analysis of the current distribution in the ITER CS-insert model coil conductor by self field measurements," MT-17 conf. 